\title{
Design of Multi-vehicle Following Control System based on Arduino
}

\author{
KeBoWen Zhang \\ Shanghai University of \\ Engineering Science \\ Songjiang Shanghai \\ 201620, China
}

\author{
Chen Deng* \\ Shanghai University of \\ Engineering Science \\ Songjiang Shanghai \\ 201620, China
}

\author{
YuLin Ou \\ Shanghai University of \\ Engineering Science \\ Songjiang Shanghai \\ 201620, China
}

\begin{abstract}
To Design multi-vehicle following control system based on Arduino, we use modularization to design wireless communication, modular infrared eye, hardware motor drive module and other hardware modules. The vehicle self organizing wireless communication network is designed by using XBEE in order to achieve the centralized control of the host computer and complete the adjustment of car speed, vehicle spacing. Use infrared ommateum to complete multi cars following and implement rapidly the vertical line operation mode. [1]
\end{abstract}

\section{General Terms}

Data Acquisition

\section{Keywords}

LabVIEW; infrared ommateum; ZigBee; intelligent car control; Queue follower.

\section{INTRODUCTION}

With the increasing of popularization of vehicles in China,the conditions of road traffic congestion are increasing as well.The traffic accidents caused by occupying lanes or outcontrolling of flow rate of traffic forts and so on happen constantly.This situation concerns traffic administrative department.The problem of traffic congestion becoming one of the critical problems in progress of urbanization, which desire to be solved.

In platform of Aduino, based on LabVIEW data acquisition and analysis system,design of ( system) could efficiently shorten the develop cycle,reduce the cost of research.So that we could achieve the aim that collect date in real time,make it interface friendly,easier to expand free wiring and have maintenance,especially its good portability and compatibility of compile program under the $\mathrm{C}$ programming, what is more,the match of various detection sensor and wireless communication equipment will be more convenience and quicker. The system apply ZigBee build wireless network control mode,actualize the control of intelligent vehicles speed,vehicle distance adjustment and vehicle information exchange. At the same time, the use of infrared compound eye sensor, multi range infrared receiver, 0 to 180 DEG in the direction of adjustment to overcome the problem that infrared line emission can not bypass.Meanwhile,it provides a new solution to actualize the rapid formation of road traffic congestion condition and keep the smooth traffic flow. [2]

\section{THE PROJECT DESIGN}

Multi intelligent car formation control system to ues ZigBee module networking which constitute a centralized control structure and use infrared module to form a distributed control structure. There are no centralized control unit and the smart car relationship is equal which are able to communicate with other cars through communication to coordinate their actions. The smart car has a high degree of autonomy. They have autonomous planning and decision making. The hardware are mainly formed by the motor drive module, infrared eye sensor module, ZigBee module. ZigBee mainly to complete the formation of the network. Through the PC release information, smart car can be completed to send and receive and realize the adjustment of the corresponding operation mode, adjust the speed, adjust the distance between the vehicle and so on. Four wheels are driven by DC motor which use differential control to achieve the car straight, turn left, turn right, back, turning left, turning right, a variety of sports. Infrared emission device using 180 to 0 degrees .Reception of infrared signals by infrared receiving infrared signal,and the receiving range at 0 to 180 degrees. According to the strength of the received signal, the car will be transmitted to the LabVIEW core processor to determine the signal in order to drive the motor drive mechanism to achieve the adjustment of the movement, and then complete the car follow the action. The specific design includes: software design, hardware design of two parts. [3]

\subsection{Software Design}

The software design includes the following three parts: Infrared programming, ZigBee system software design and LabVIEW host computer control software design. The IR program design includes: the infrared emission infrared eye program design and program design.

\subsection{1 infrared design}

1) infrared emission program design

Infrared emission has only two states. Open the launching state, then car tail is 0 to 180 degrees outward radiation of infrared signal and wait for the car after receiving ommateum.

\section{2) Program Design of Infrared Compound Eye}

Once on the silence state of wireless network, the vehicle begins to complete the following control, the primary task of which is to finish the following action of the multi-intelligent vehicle. First, in the initialization state, the vehicle keeps by default the linear following state. Right on the completion of the initialization, the vehicle goes into the period of angle detection by the compound eye, judging the motor drive module according to the angle. The whole process goes repeatedly until the finish of the vertical queuing by all vehicles, which then move forward straightly in uniformed speed. If there is a stop signal, the vehicle would enter the stop state, ready to receive information. The process is shown in Fig.1. [4] 


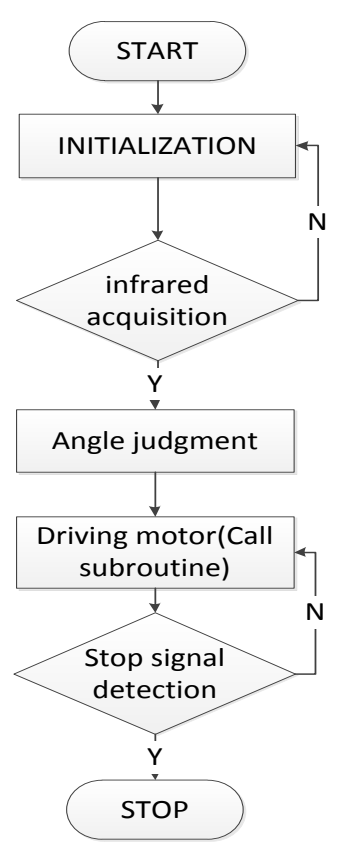

Fig 1 Infrared flow chart

\subsubsection{The Design of ZigBee System Software}

The general designed structure of ZigBee system software consists of a coordinator and a router (See Fig.2), The routing node of ZigBee sends the collected data to the coordinator node by the form of multi-hop and is responsible, meanwhile, for the relaying and forwarding of data; The coordinator node of ZigBee is responsible for the start of Zig Bee network, address configuration of network members and so on. The collected data from ZigBee network is, then be sent to the upper computer for display through serial ports

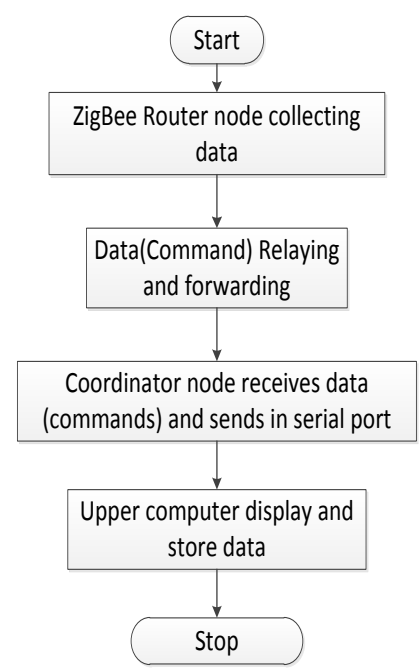

Fig 2 Zigbee software design flow chart

\subsubsection{The Control Software Design of LabVIEW's Upper Computer}

As can be seen from above, the child nodes, once entering ZigBee network, need to coordinate with the control software of the upper computer, waiting and receiving command codes from the control software like the system in-place, start command and so on. Test data of all child nodes is collected by ZigBee network before sent to the LabVIEW control program for further processing. Finally, the data is stored, analyzed in LabVIEW before the final processing result is shown.

The processing program of the upper computer is shown in Fig.3. [5]

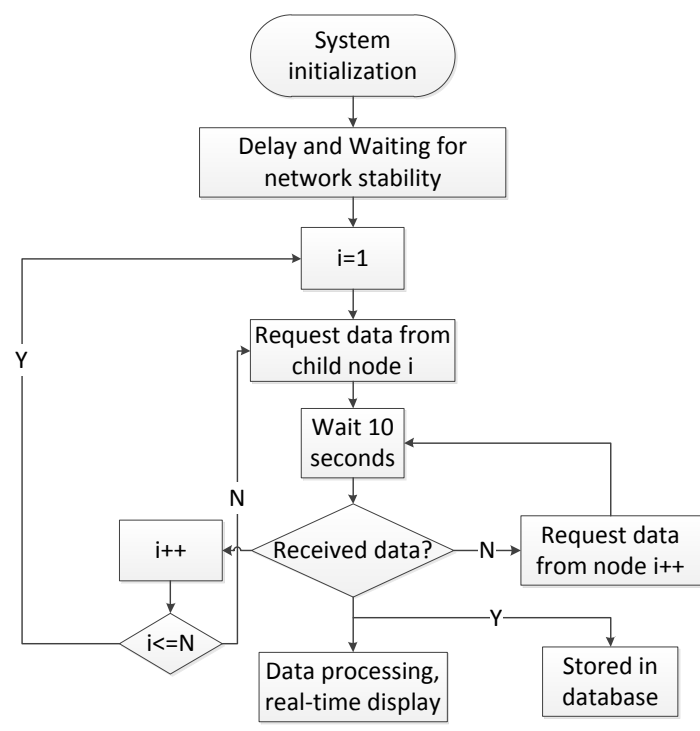

Fig 3 Process flow chart of the upper computer

\subsection{Hardware Design}

Based on Arduino, the multi-vehicle following control system is composed of the motor drive module, power voltagestabilizing circuit, ZigBee communication module, Arduino microprocessor control circuit and sensor detection system. The connection between the motor drive module and the power voltage-stabilizing circuit is assisted through net interface, while the Arduino microprocessor is connected with the sensor detection system through the A/D interface. The Arduino microprocessor, sends the control signal based on data received from the sensor detection system, and change the speed and steering of DC motors at the left and right side by controlling the motor drive module. The hardware of the intelligent vehicle, based on the concept of module design, mainly consists of the following modules: 1) Arduino--UNO core processor 2) infrared sensor module 3) ZigBee communication module 4) motor drive module. The structure of the hardware is shown in Fig.4. [6]

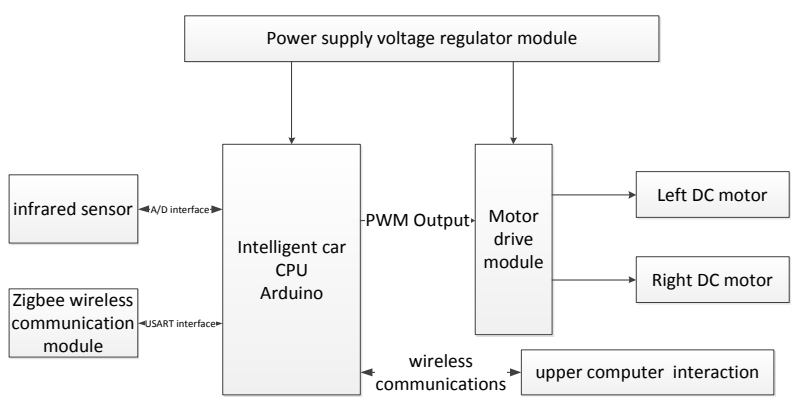

Fig 4 hardware structure chart

\subsubsection{The Motor Drive Module and Peripheral}

\section{Interface Design}

Motor drive module comprising: L298N motor driver chip, 74HC00 chip and DC motor.Corresponding to (1)(2)(3) in Fig. 5. Among them (1) can achieve control two DC motors,but also has control enable. Which operating voltage input $7.5 \mathrm{~V}$, $5 \mathrm{~V}$ from pins 6,12 , and ENA, ENB is active-high enable, 
respectively IN1 and IN2, IN3 and IN4. Pins 4, 5 and 16, 17 respectively connected with a motor,and pins 7,9,13,15 are connected to the input level,which can control motor reversing. (2) 74HC00 chip to achieve that the D4, D7 level emitted and the NOT gate handle were sent to pins 7 and 9, 13 and 15. (3) can achieve that control the rotation of the motor M1 and M3, M4 and M2 by output level from pins 4 and 5, 16 and 17 and jumper $\mathrm{J} 2, \mathrm{~J} 3$ at the same time. [7]

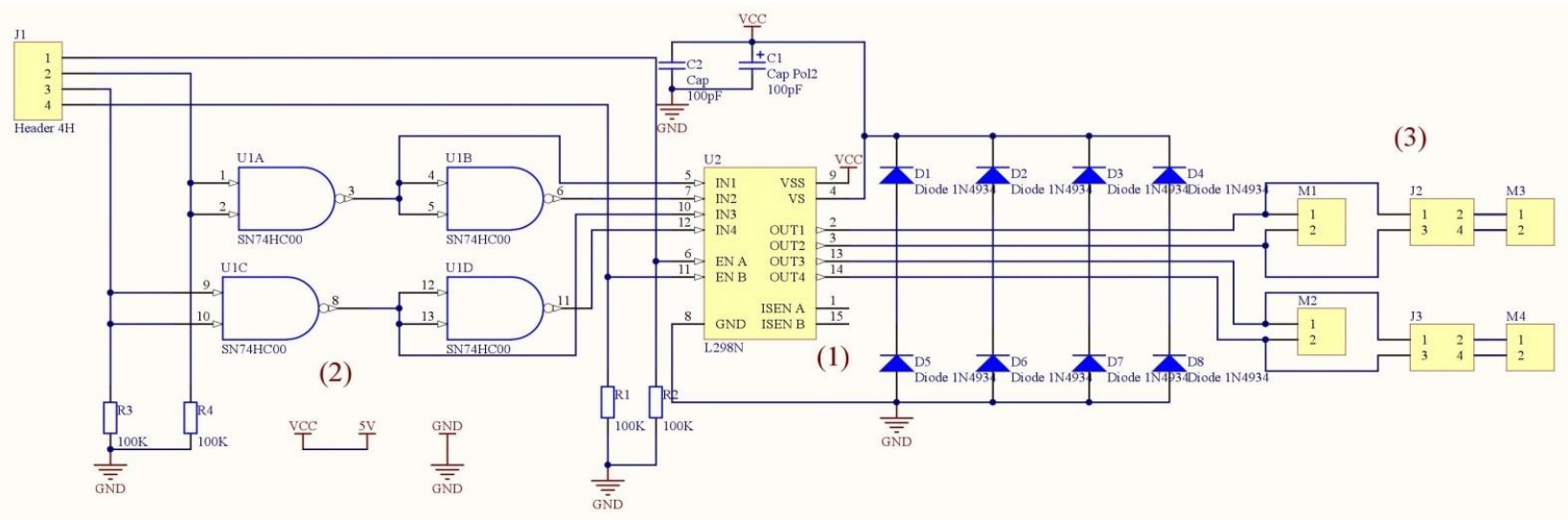

Fig. 5 Connection Diagram of Motor Drive Module

\subsubsection{Communication Module of ZigBee}

1 、 Localization Method:The protocol of Zigbee is IEEE802.15.4, which is one standard of IEEE Wireless personal area network(WPAN).IEEE802.15.4 standard only defines two kinds of service:Physical layer(PHY) and Media Access Control layer(MAC).Zigbee Alliance establish Network layer(NWK) and the architect of applications based on these.Application layer is consist of Application Support Layer(APS) and Zigbee Device Object(ZDO).The physical layer can apply to two different frequency range: $868 / 915 \mathrm{MHz}$ and $2.4 \mathrm{GHz} .868 \mathrm{MHz}$ is used in Europe, and $915 \mathrm{MHz}$ is used in United State and Australia.The Media Access Control layer control the access of wireless channel by CSMA-CA.

2、 System's feature:This intelligent car system is based on Zigbee wireless network technology to transmit the information.The data of traffic is rely on IR sensor,image taken by cameras and collection and analysis technologies. The reveal of the instruction is showed by the LED/LCD which connects the UART with Zigbee.This method is more efficient and requires less hardware.

3、 The principle of collecting data:Normally,Zigbee network is consist of three nodes:Coordinator node,router node,sensor node.The coordinator is used to constitute a Zigbee network and allot the address to the nodes added in the network at first.Every network only need one coordinator,and the router is the full-featured node which can emit and receive the data.

4、Application:Our research of vehicle's formation control choose the smart car model based on Zigbee wireless network instead of the real vehicles, which has the advantage of more stable communication,lower cost and more convenient in maintain.This way is more convenient for these kind of researches in formation control,cooperation,car to car communication and algorithms of formation. [8]

\section{DESIGN AND IMPLEMENTATION}

The most important thing is the development of infrared sensor,for adjusting the power of infrared emission is very important, which effects the quality of the signal,interference factors and so on. We utilize sliding rheostat, NPN triode to adjust the power to the most appropriate state.In the end,we decide to parallel the resistor with $51 \Omega$ in the circuit,which can enable the infrared of smart car detects the largest distance within 1 meter.
Secondely,the fabrication of annular infrared emission and receiver circuit fulfill the function of keeping emission and receiver of infrared signal with range from 0 to 180 .the main purpose in this section is to finish the detection of analog signal by using infrared sensor.According to the strength of signal locate the front smart car's direction.then change the smart car's move.At the same time,the framework of XBEE wireless network with the star model or topology network makes all the smart car(terminal) in the intelligent car system communicate with each other by router.The most important thing is that utilizing Zigbee network can control every terminals

Zigbee technology is a kind of new wireless network technology resemble the Radio Frequency Identificati(RFID) and Bluetooth,which is mainly used in short-range wireless connection and communication.The protocol is according to the standard of 802.15.4,the radio waves transmit data from one sensor to another sensor,making different sensors can communicate with each other.In the Zigbee sensor network,the location of the every component is vital for the monitoring activities. The location of the network node can calculate by location algorithm .

Finally,the debug result of two smart cars can also extend in multi-vehicle system's formation control.Great amount of test data and process of adjustment prove that the adjustment of the smart car's speed has considerable influence to the whole intelligent system.Only keep the speed of the smart cars in proper state can realize the multi-vehicle formation control system. [9]

\section{CONCLUSION}

This paper start from the analysis of the demand of multivehicle following control system,using ZigBee wireless communication network, to realize information interaction of vehicle-vehicle communication mode. Smart car with infrared navigation,Arduino--UNO as the core processor, LabVIEW as the upper computer. The hardware adopts module design idea, design the ZigBee communication module, infrared sensor, motor driver module design the formation of multi intelligent car following control application. In the laboratory environment, the start and stop of the multi intelligent vehicle formation, the queue following and the angle adjustment control task are realized. However, the system is still inadequate, the system is designed to complete the multi car 
follow the action and the simple interaction of information, the car in the choice of mobile location and speed of the best choice for further research. [10]

\section{REFERENCES}

[1] Yang M, Parent M. Cybernetic technologies for cars in Chinese cities [C] // Proceedings of City Trans China . Shanghai, China, 2004:17-18.Tao, G., Kokotovic,P. V. Adaptive control of systems with backlash. Automatica, 1993,29(2):323-335.

[2] Li Nan Zu, Tian Yan Tao, Hao Mei. Large scale multi mobile robot cooperative task of distribution, autonomy and cooperation system [J]. Robot, 2006, 28 (5). 470477

[3] http//www.maxstream.net/products/xbee/product manual_XBee_OEM_RF_Modules.pdf.

[4] Zhai Lei, Liu Shengde, Hu Xianbin. ZigBee technology and application $[\mathrm{M}]$. Beijing: Beihang University press, 2007

[5] Zhuo Qing, Huang Kaisheng, Shao Beibei et al. Learn to do smart car $[\mathrm{M}]$. Beijing: Beihang University press, 2007
[6] Yang Ming, Cheng Lei, Huang Weihua et al. Application of steering gear control of intelligent vehicle based on photoelectric tube tracking [J]., 2007, 22 (1): 50-53.

[7] Wu Jianping, yin and the Warring States, Cao Sirong et al. Application of infrared reflective sensor in the navigation of autonomous tracking vehicle $[\mathrm{J}]$. China test technology, 2004,30 (6): 21-23.

[8] Banzi M . Getting Started with Arduino [M]. USA: O’Reilly Media, 2008.

[9] Yang Jizhi, Yang Yuhuan. Arduino network interactive product innovation design [J]. electrical and mechanical product development, based on the 2012, 25 (1): 99 100

[10] LORSCHEITER, Aguirre T, PAIM, et al. Using Ms-Visual studio and Arduino to do temperature measurements[J]. Periodico tche Quimica, 2011, 8 (16) : $60-66$. 